\title{
Inequalities in access to bariatric surgery in Canada
}

\author{
Arya M. Sharma MD
}

See also CMAJ Open article www.cmajopen.ca/content/3/3/E331

$\mathrm{R}$ ecent analyses show a disproportionate growth in the number of Canadians living with class II (body mass index [BMI] 35.0-39.9) and class III (BMI $\geq 40.0$ ) obesity conditions that are expected to affect $6 \%$ of adult Canadians (about 2 million people) by $2019 .{ }^{1}$ Although its cause appears to be simple (positive caloric balance), obesity is a complex heterogeneous disorder driven by genetic, environmental, metabolic and behavioural factors. Once excess weight is established, volitional efforts to induce and sustain lower energy balance (e.g., by restricting caloric intake, increasing caloric expenditure or both) result in a complex and persistent neuroendocrine response that limits the magnitude of weight loss and ultimately restores body weight. ${ }^{2}$ For this reason, an increasing number of organizations, including the Canadian Medical Association, World Health Organization, American Medical Association and US Food and Drug Administration, now consider obesity a chronic disease.

Sustained weight loss with behavioural interventions seldom exceeds 3\%-5\% of initial body weight. ${ }^{3}$ Emerging pharmacotherapies promise to boost sustainable weight loss (with ongoing treatment) to the $5 \%-12 \%$ range $^{4}$ but have yet to become widely available. With appropriate patient selection, education and follow-up, bariatric surgery can offer sustainable weight loss in the 20\%-30\% range, with substantial reductions in morbidity and mortality and marked improvements in mental health and quality of life. ${ }^{5}$

According to a recent report by the Canadian Institute for Health Information, ${ }^{6} 6525$ bariatric surgeries were done across Canada in the 2013/14 fiscal year, a $313 \%$ increase from 2006/07. However, the availability of this treatment remains severely restricted, with substantial inequalities in access between provinces (Figure 1) and no access in Prince Edward Island or the territories. Annual access to bariatric surgery (2012/13) per 1000 people living with a BMI of more than $35 \mathrm{~kg} / \mathrm{m}^{2}$ (2007-2010 prevalence) in Canada is about 5.4; ${ }^{6}$ however, this number ranges from 9.6 in Quebec to 1.1 in Nova Scotia - an almost 10 -fold difference. To catch up with the current rate of surgery in Quebec, Alberta would need to perform an additional 813 procedures each year, British Columbia would need an additional 805 and Nova Scotia would need an additional 463. Overall, bringing the rate of surgery across Canada to the current rate in Quebec would require an additional 5129 surgeries per year. However, there are currently no accepted benchmarks for the required number of bariatric surgeries that would be needed to achieve a meaningful reduction in the burden of severe obesity in Canada.

Disparity in bariatric surgery across Canada likely reflects varying priorities and policies between provinces. For example, higher surgery rates in Quebec may be the result of the pioneering work in bariatric surgery at Laval and McGill universities. In contrast, a more recent surge in bariatric surgeries in Ontario with a $\$ 75$ million investment in the Ontario Bariatric Network was largely driven by the urgent need to repatriate the rising number of Ontario residents who leave the province to receive surgery in the United States, with the costs of the operation for these patients covered by the Ontario Health Insurance Plan (1660 in fiscal year 2008/09). ${ }^{7}$ Despite this increase, restricted access to bariatric surgery across Canada still results in wait times that may exceed several years, with substantial health and economic consequences for patients living with severe obesity. ${ }^{6}$

Given that the benefits and cost-effectiveness of bariatric surgery are well established, one can only speculate as to why provincial health authorities are not scrambling to further increase access to this treatment. Although it is important to acknowledge that obesity competes for scarce resources and that choices must be made as to

\section{KEY POINTS}

- Fewer than 6 out of 1000 Canadians living with class II or class III obesity receive a bariatric surgery procedure each year, with substantial disparities in rates between provinces.

- An estimated yearly increase of 5129 surgeries is required to align the national rate with that of Quebec.

- Better infrastructure for the provision of bariatric surgery should be integrated into a comprehensive national obesity management strategy. 
what services and treatments to provide, care must be taken to ensure that access to obesity surgery is not restricted based on the perception that it provides patients with "an easy way out." Indeed, patients living with obesity continue to face widespread stigma and discrimination within the health care system. The "blame and shame" approach to managing obesity continues to find supporters despite evidence pointing to the ineffective and counterproductive effects of this strategy, which serves only to threaten health, generate health disparities and interfere with effective intervention efforts. ${ }^{9}$

Even with a substantial increase in funding for bariatric surgery, Canadian health care systems would struggle to meet the potential demand owing to the lack of infrastructure and trained health care professionals. A triage system that ensures patients in the greatest need receive treatment first is required. Unfortunately, the current BMI-based criteria do not fulfill this need. Although BMI is helpful as a population measure of obesity, when applied to an individual, it fails to reliably reflect the actual health or functional status of the person. This shortfall has led to the development of the Edmonton Obesity Staging System, which ranks the mental, medical and functional health of patients with obesity on a five-point ordinal scale

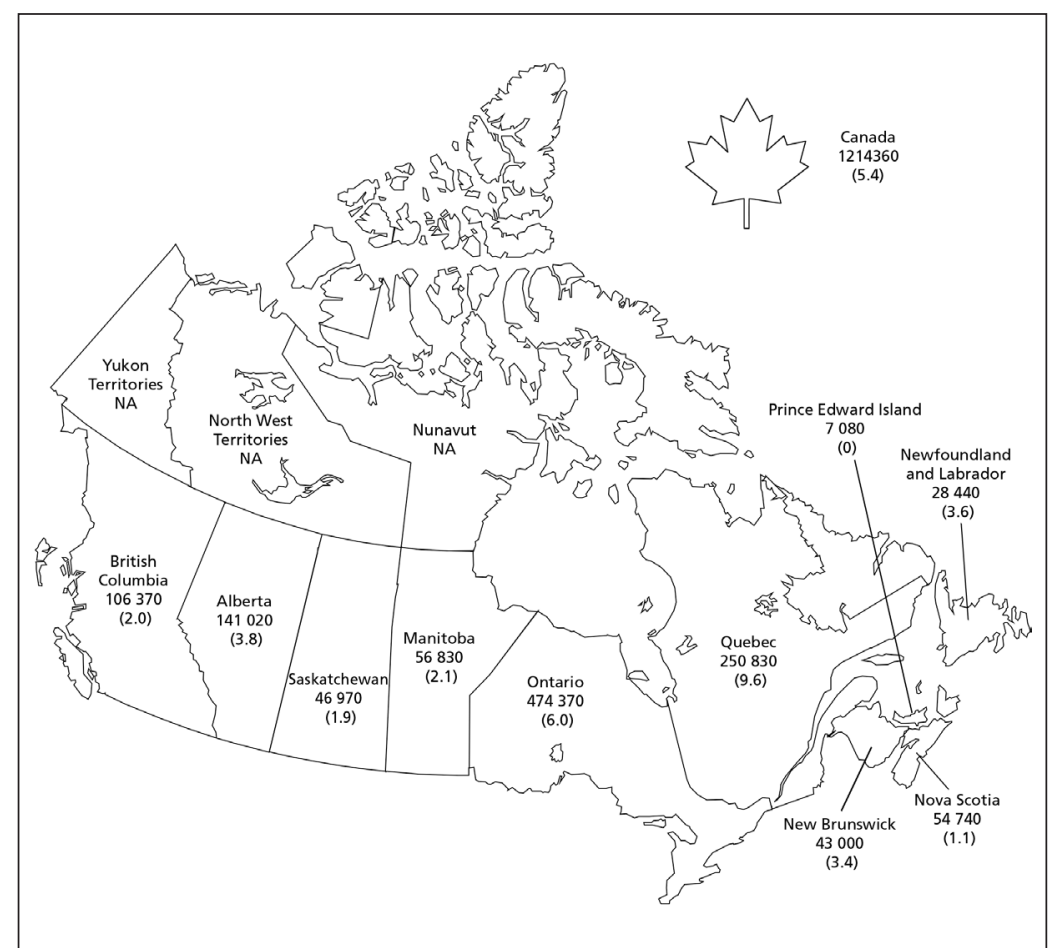

Figure 1: Prevalence of obesity and (in parentheses) rates of bariatric surgeries performed per 1000 adults with class II or III obesity in 2013/14, by province and (top right) for Canada overall. ${ }^{6}$ ranging from stage 0 (no mental, medical or functional limitations) to 4 (severe mental, medical or functional limitations). ${ }^{10}$ This system is a better predictor of mortality than BMI or other anthropometric measures of obesity and could provide a more meaningful means of triaging patients than BMI alone.

Other aspects of bariatric care cannot be ignored. Efforts at secondary prevention to reduce and limit weight gain in patients already carrying excess weight must be increased. Given the more than 6 million Canadians living with obesity, these services must be provided at the primary care level rather than at specialized centres. The established tenets of chronic disease management, which include patient education, self-management and ongoing follow-up and support - the principles that are embedded in the " 5 As of Obesity Management" developed by the Canadian Obesity Network — should be applied. ${ }^{11}$ Finally, education on the complex causes and evidence-based management of obesity needs to be integrated into every level of professional education for physicians and allied health professionals.

\section{References}

1. Twells LK, Gregory DM, Reddigan J, et al. Current and predicted prevalence of obesity in Canada: a trend analysis. CMAJ Open 2014;2:E18-26.

2. Sumithran P, Prendergast LA, Delbridge E, et al. Long-term persistence of hormonal adaptations to weight loss. $N$ Engl $J$ Med 2011;365:1597-604.

3. Franz MJ, van Wormer J, Crain AL, et al. Weight-loss outcomes: a systematic review and meta-analysis of weight-loss clinical trials with a minimum 1-year follow-up. J Am Diet Assoc 2007; 107:1755-67.

4. Rueda-Clausen CF, Padwal RS, Sharma AM. New pharmacological approaches for obesity management. Nat Rev Endocrinol 2013;9:467-78.

5. Courcoulas AP, Yanovski SZ, Bonds D, et al. Long-term outcomes of bariatric surgery: a National Institutes of Health symposium. JAMA Surg 2014;149:1323-9.

6. Bariatric surgery in Canada [report]. Ottawa: Canadian Institute for Health Information; 2014. Available: www.cihi.ca/CIHI -ext-portal/xlsx/internet/bariatric_data_051415_en (accessed 2015 Oct. 5).

7. Born K, Park S, Laupacis A. Need \& access to bariatric surgery in Canada. Healthydebate.ca; 2011 Aug. 17. Available: http:// healthydebate.ca/2011/08/topic/cost-of-care/bariatric-surgery (accessed 2015 Oct. 5).

8. Vartanian LR, Fardouly J. The stigma of obesity surgery: negative evaluations based on weight loss history. Obes Surg 2013; 23:1545-50.

9. Puhl RM, Heuer CA. Obesity stigma: important considerations for public health. Am J Public Health 2010;100:1019-28.

10. Padwal RS, Pajewski NM, Allison DB, et al. Using the Edmonton obesity staging system to predict mortality in a population-representative cohort of people with overweight and obesity. CMAJ 2011;183:E1059-66.

11. Rueda-Clausen CF, Benterud E, Bond T, et al. Effect of implementing the $5 \mathrm{As}$ of obesity management framework on provider-patient interactions in primary care. Clin Obes 2014; 4:39-44.

Affiliation: Division of Endocrinology, Department of Medicine, Faculty of Medicine and Dentistry, University of Alberta, Edmonton, Alta. 